\title{
In vivo evaluation of poly-L-asparagine nanocapsules as carriers for anti-cancer drug delivery
}

\author{
Gustavo R. Rivera-Rodriguez ${ }^{\mathrm{a}, \mathrm{b}, 1}$, Giovanna Lollo ${ }^{\mathrm{a}, \mathrm{b}, \mathrm{c}}$, Tristan Montier ${ }^{\mathrm{c}, \mathrm{d}}$, \\ Jean Pierre Benoit ${ }^{\mathrm{d}, \mathrm{e}}$, Catherine Passirani ${ }^{\mathrm{d}, \mathrm{e}}$, Maria José Alonso ${ }^{\mathrm{a}, \mathrm{b}}$, Dolores Torres ${ }^{\mathrm{b}, *}$ \\ ${ }^{a}$ Center for Research in Molecular Medicine and Chronic Diseases (CIMUS), Campus Vida, University of Santiago de Compostela, Health Research Institute \\ of Santiago de Compostela (IDIS), 15706 Santiago de Compostela, Spain \\ b Department of Pharmacy and Pharmaceutical Technology, School of Pharmacy, Campus Vida, University of Santiago de Compostela, 15782 Santiago de \\ Compostela, Spain \\ ' INSERM UMR_S 1066, Université d'Angers, Angers, France \\ d INSERM U1078 - UBO, EFS Bretagne, France \\ e LUNAM Université, Université d'Angers, Angers, France
}

\section{A R T I C L E I N F O}

\section{Article history:}

Received 20 July 2013

Received in revised form

26 September 2013

Accepted 29 September 2013

Available online 17 October 2013

\section{Keywords:}

Polyaminoacids

Poly-L-asparagine

Nanocapsules

Docetaxel

Cancer therapy

Tumor targeting

\begin{abstract}
A B S T R A C T
Here, we report the in vivo proof of-concept of a novel nanocarrier, poly-L-asparagine (PASN) nanocapsules, as an anticancer targeted drug delivery system. The nanocapsules were loaded with the fluorescent marker DiD (1,1'-dioctadecyl-3,3,3',3'-tetramethylindodicarbocyanine perchlorate) and also with the model drug docetaxel to evaluate the biodistribution and efficacy profiles in healthy and glioma-bearing mice, respectively. Regardless of their cargo, the nanocapsules presented a size close to $180 \mathrm{~nm}$, a surface charge around $-40 \mathrm{mV}$ and an encapsulation efficiency of $75-90 \%$. The biodistribution study in healthy mice showed that PASN nanocapsules led to a two- and three-fold increment in the mean residence time (MRT) and area under the curve (AUC) values, respectively, compared to those of a non-polymeric nanoemulsion. Finally, the efficacy/toxicity study indicated that the encapsulated drug was as efficacious as the commercial formulation (Taxotere ${ }^{\circledR}$ ), with the additional advantage of being considerably less toxic. Overall, these results suggest the potential of PASN nanocapsules as drug nanocarriers in anticancer therapy.
\end{abstract}

(C) 2013 Elsevier B.V. All rights reserved.

\section{Introduction}

The design of targeted anticancer drug delivery nanocarriers receives a great deal of attention recently. These efforts have so far resulted in the development of a variety of drug nanocarriers (Alonso and Couvreur, 2012) and, more importantly, the clinical development and marketing of a significant number of anticancer nanomedicines (Li et al., 2012; Wang et al., 2012). However, despite these advances, it is broadly accepted that further improvements in nanotechnologies are required in order to obtain more selective targeting to cancer cells (Yu et al., 2012).

Among the nanocarriers described so far, nanocapsules, consisting of an oily core surrounded by a polymer shell represent a promising option justified by their versatility (Hervella et al., 2008; Garcion et al., 2006; Wadhwa et al., 2012). Briefly, the shell of

\footnotetext{
* Corresponding author. Tel.: +34 881814880 .

E-mail address: dolores.torres@usc.es (D. Torres).

1 Present address: IBBP, University of Münster, Schlossplatz 8, 48143 Münster, Germany.
}

the nanocapsules has been designed to confer long circulating and potential targeting properties, whereas the core, because of its oily nature, can accommodate great amounts of lipophilic drugs.

Despite the multiple advantages of nanocarriers, historically, the great formulation challenge, prior to targeting, has been to avoid their immediate uptake by the mononuclear phagocyte system (MPS) and, thus, their rapid elimination after intravenous administration. The main strategy used, so far, has been mainly the use of hydrophilic polymers as coatings, commonly polyethylene glycol (PEG) (Huynh et al., 2010). However, it is well known that other polymers, such as polysaccharides (Liu et al., 2008) and poly (amino acids) (Romberg et al., 2007a), provide similar advantages. Within this frame, poly-L-asparagine (PASN) is, in our view, an attractive biomaterial that could be used as a hydrophilic shell for nanocarriers, the same way as it has been described for liposomes (Metselaar et al., 2003; Romberg et al., 2006). Actually, besides the long circulating properties achieved by PASN, it has the advantages of being degraded by the lysosomal proteases (Romberg et al., 2007a) and brings targeting characteristics of the carriers toward malignant cells. In this way, it is known that the amino acid L-asparagine (ASN), an essential nutrient for protein 
synthesis, despite being produced in sufficient amounts by normal cells, in cancer cells the amount synthesized does not fulfill the higher tumor cell requirements in order to keep up their sprawl (Verma et al., 2007; Chabner and Friedmann, 2006), the ASN has become a needful aim in cell diet. Thereby, taking advantage of the avidity of cancer cells by ASN, we have hypothesized that PASN might be a valuable biomaterial for the targeted delivery of anticancer drugs. From this background, our group has recently reported the development of a new type of nanocapsules coated with the poly-amino acid PASN aimed at targeting antitumor drugs to cancer tissues (Rivera-Rodriguez et al., 2013).

In this way, the main purpose of the current study was to evaluate the potential of these anticancer drug delivery systems in an in vivo model. Particularly, the biodistribution and plasma kinetic profiles has been analyzed through imaging and analytical techniques, as well as the antitumoral effect of these nanocapsules after single intravenous injection given to glioma-model-bearing nude mice.

\section{Materials and methods}

\subsection{Chemicals}

Docetaxel $\geq 97 \%$ (DCX; from Fluka), Poloxamer 188 (Pluronic $^{\circledR}$ F68), benzalkonium chloride (BKC) and poly-L-asparagine, $15 \mathrm{kDa}$, (PASN) were purchased from Sigma-Aldrich (Spain). Miglyol ${ }^{\circledR} 812$, neutral oil formed by esters of caprylic and capric fatty acids and glycerol, was kindly provided by Cremer Oleo GmbH \& Co. (Germany). The surfactant Epikuron ${ }^{\circledR} 170$, a phosphatidylcholine enriched fraction of soybean lecithin, was donated by Cargill (Spain). 1,1'-Dioctadecyl-3,3,3',3'-tetramethylindodicarbocyanine perchlorate (DiD) was obtained from Molecular Probes-Invitrogen (USA). Taxotere ${ }^{\circledR}$ was provided by the Hospital Pharmacy of Angers.

\subsection{Preparation of PASN nanocapsules}

PASN nanocapsules were obtained by the solvent displacement technique (Calvo et al., 1997), recently adapted by our group (Oyarzun-Ampuero et al., 2013; Lozano et al., 2008). Briefly, an organic phase, composed of $0.5 \mathrm{ml}$ of ethanol $30 \mathrm{mg}$ of Epikuron ${ }^{\circledR}$ $170,150 \mu \mathrm{l}$ of Miglyol ${ }^{\circledR} 812$ and $9 \mathrm{ml}$ of acetone containing $4 \mathrm{mg}$ of BKC, was poured into $20 \mathrm{ml}$ of aqueous phase containing $50 \mathrm{mg}$ of poloxamer 188 and $10 \mathrm{mg}$ of PASN under mild stirring. Finally, solvents were eliminated from the suspension under vacuum to a constant volume of $5 \mathrm{ml}$. The DiD and DCX-loaded nanocapsules were obtained by replacing the $0.5 \mathrm{ml}$ of ethanol in the organic phase with $0.5 \mathrm{ml}$ of an ethanol solution containing the drug. The concentrations of these ethanol solutions were $20 \mathrm{mg} / \mathrm{ml}$ and $1 \mathrm{mg} / \mathrm{ml}$ of DCX and DiD, respectively.

Two different nanoemulsions, with anionic and cationic surfaces, were prepared and used as controls. Both nanoemulsions were prepared following the above described procedure. However, for the anionic nanoemulsion, the cationic surfactant and the polymer PASN were omitted; whereas for the cationic nanoemulsion only the PASN was omitted.

\subsection{Characterization of PASN nanocapsules}

PASN nanocapsules were characterized regarding their size, zeta potential and morphology as follows:

Particle size and polydispersity index were determined by Dynamic Light Scattering after dilution of nanocapsules in $\mathrm{KCl}$ $1 \mathrm{mM}, 1: 100(\mathrm{v} / \mathrm{v})$. Each analysis was carried out at $25^{\circ} \mathrm{C}$ with an angle detection of $173^{\circ}$. The zeta potential values were determined by laser Doppler anemometry in the same dilution and temperature conditions. Particle size and zeta potential analysis were performed in triplicate using a zetasizer NanoZS ${ }^{\circledR}$ (Malvern Instruments, Malvern, UK).

\subsection{DCX encapsulation efficiency}

DCX encapsulation efficiency of PASN nanocapsules was determined indirectly. For this purpose the non-encapsulated DCX, obtained from the infranatant of ultracentrifuged nanocapsules $\left(30,000 \times g, 1 \mathrm{~h}, 4^{\circ} \mathrm{C}\right)$, was quantified by HPLC. The sample obtained from clear infranatant, was diluted with acetonitrile and centrifuged $(20 \mathrm{~min}$ at $4000 \times \mathrm{g})$; the supernatant was injected into the HPLC system for analysis. As complementary control, the total amount of drug was estimated from aliquots of non-isolated PASN nanocapsules dissolved in acetonitrile.

The DCX analytical method used was a slightly modified version of that proposed by Lee et al. (Lee et al., 1999). The HPLC system consisted of an Agilent 1100 Series HPLC instrument equipped with a UV detector, $\lambda=227 \mathrm{~nm}$, and a reverse phase Zorbax Eclipse ${ }^{\circledR}$ XDB-C8 column $(4.6 \mathrm{~mm} \times 150 \mathrm{~mm}$ of internal diameter, pore size $5 \mu \mathrm{m}$; Agilent USA). The mobile phase consisted of a mixture of acetonitrile and $0.1 \%(\mathrm{v} / \mathrm{v})$ ortho-phosphoric acid $(55: 45, \mathrm{v} / \mathrm{v})$ with a constant flow rate of $1 \mathrm{ml} / \mathrm{min}$.

The encapsulation efficiency (EE) for DCX was calculated as follows:

$\mathrm{EE}(\%)=\frac{(A-B)}{A} \times 100$

where $A$ is the experimental total drug concentration and $B$ is the drug concentration quantified in the clear infranatant after nanocapsule separation, corresponding to unloaded drug.

\subsection{Encapsulation efficiency of fluorescent probe DiD, into the nanocapsules}

The encapsulation efficiency of DiD in PASN nanocapsules was also determined indirectly using an UV spectrophotometric method, at $\lambda=646 \mathrm{~nm}$. The total amount of drug was estimated by dissolving an aliquot of non-isolated DiD-loaded PASN nanocapsules with acetonitrile. This sample was centrifuged during $20 \mathrm{~min}$ at $4000 \times g$, achieving the precipitation of the hydrophilic compounds, the supernatant was measured. The non-encapsulated drug was determined by the same method following separation of the PASN nanocapsules from the aqueous medium by ultracentrifugation at $20,000 \times g$ for $1 \mathrm{~h}$ at $15^{\circ} \mathrm{C}$.

\subsection{Hemolysis study}

\subsubsection{Preparation of red blood cells (RBC) suspension}

The blood of female Wistar rats was obtained by cardiac puncture and then placed in a tube containing heparin. The $2 \%(\mathrm{w} / \mathrm{v})$ suspension of red blood cells (RBCs) was prepared as follows. First, the blood with heparin was centrifuged at $600 \times \mathrm{g}$ at $4{ }^{\circ} \mathrm{C}$ during $15 \mathrm{~min}$; then, the plasma and the top $2-3 \mathrm{~mm}$ of the pellet was removed. The pellet was resuspended in PBS $(\mathrm{pH} 7.4)$ previously chilled to $4{ }^{\circ} \mathrm{C}$. The suspension was then centrifuged as indicated above. This washing process was repeated three times. The final RBCs concentration of the reconstituted pellet was $2 \%(\mathrm{w} / \mathrm{v})$ in PBS solution ( $\mathrm{pH} 7.4)$. Finally, the RBC suspension was stored at $4{ }^{\circ} \mathrm{C}$.

\subsubsection{Lysis quantification}

A series of nanocapsules dilutions were prepared in PBS, pH 7.4. Dilutions of each concentration were mixed with the same volume of $2 \%(w / v)$ RBC suspension. The $\mathrm{pH}$ of each solution was adjusted to 7.4 by adding $\mathrm{HCl}$ and then incubated for $30 \mathrm{~min}$ at $37^{\circ} \mathrm{C}$. Each solution was centrifuged at $600 \times \mathrm{g}$ and $4{ }^{\circ} \mathrm{C}$ for $15 \mathrm{~min}$ and the 
concentration of the released hemoglobin in the supernatant was measured by spectrophotometry at $\lambda=550 \mathrm{~nm}$. Triton $\mathrm{X}-100(1 \%$, $\mathrm{v} / \mathrm{v}$ ) and PBS (pH 7.4), were used to set the two reference hemolysis values, $100 \%$ and $0 \%$, respectively. Hemolysis percentage was calculated as follows:

RBCs lysis $(\%)=\left[\frac{A_{\mathrm{NC}}-A_{\text {blank }}}{A_{\text {Triton X-100 }}-A_{\text {blank }}}\right] \times 100$

where $A_{\mathrm{NC}}$ is the absorbance of supernatant in nanocapsules - RBC mixture, $A_{\text {Triton X-100 }}$ is the absorbance of supernatant of Triton X100 and RBC mixture and $A_{\text {blank }}$ is the absorbance of the supernatant in PBS and RBC mixture.

\subsection{In vivo studies}

\subsubsection{Pharmacokinetic study}

Animal care was in strict accordance to French Ministry of Agriculture regulations. The dosage scheme was as follows, a single $150 \mu \mathrm{l}$ injection of fluorescent PASN nanocapsules administered in the tail vein of six-week old female Swiss mice with 20-22 g body weight (Ets Janvier, Le Genest-St-ile, France). After injection, blood samples were collected by cardiac puncture in the next times post-administration, 30, 60, 120, 180, $240 \mathrm{~min}$ and $24 \mathrm{~h}$. The collection was done by triplicate and the mixture of collected blood was centrifuged for $10 \mathrm{~min}$ at $2000 \times \mathrm{g}$ in a venous blood collection tube (Vacutainer, SST II Advance, 5 ml, Becton Dickinson France SAS, France).

Plasma-remaining fluorescence was quantified as follows: $150 \mu \mathrm{l}$ of the supernatant was placed in a black 96-well plate (Greiner Bio-one, Germany) and measured in a Fluoroscan (Ascent FL, Thermo Fischer Scientific, France) with $\lambda$-emission $=665 \mathrm{~nm}$ and $\lambda$-excitation $=644 \mathrm{~nm}$. The results were analyzed with the Ascent software for Fluoroscan (Thermo Fischer Scientific, France). The total blood volume, using density to calculate concentrations, was assumed as the $7.5 \%$ of the total body weight. DiD concentrations were expressed as fluorescence units (FUs) and each value was corrected with the FUs measured in a negative control. The negative control was considered as normal blood fluorescence, so the fluorescence of 3 blood-supernatant samples of 3 mice injected with an isotonic solution (150 $\mathrm{nM} \mathrm{NaCl}$ ) was quantified. At zero time, $100 \%$ of fluorescence was considered as simple mixture of nanocapsules and blood, maintaining the in vivo conditions of concentration.

Pharmacokinetic data were treated considering a noncompartmental analysis of the percentage of the injected dose $v s$ time. The profiles were obtained with Kinetica 5.1 software (Thermo Fischer Scientific, France). The half-lives $\left(t_{1 / 2}\right)$ were calculated as follows:

$t_{1 / 2}=\frac{\log (2)}{L z}$

The $L z$ was determined from linear regression using defined intervals, $1 \mathrm{~h}$ and $24 \mathrm{~h}$ for $t_{1 / 2}$ distribution [0-1 h] and $t_{1 / 2}$ elimination [1-24h], respectively. The trapezoidal rule was used to calculate the area under the curve (AUC) during the whole experimental period (AUC [0-24h]) without extrapolation, as well as the area under the first moment curve (AUMC). The mean residence time (MRT) was calculated from 0 to $24 \mathrm{~h}$, from the following equation:

$\operatorname{MRT}[0-24 \mathrm{~h}]=\frac{\text { AUMC }[0-24 \mathrm{~h}]}{\mathrm{AUC}[0-24 \mathrm{~h}]}$

\subsection{In vivo antitumor efficacy study}

Tumor-bearing mice were prepared by injecting subcutaneously a suspension of $1 \times 10^{6}$ cells of U87MG glioma line
(ATCC, Manassas, VA) in $150 \mu$ l of Hanks Balanced Saline Solution (HBSS) into the right flank of athymic nude mice (6 weeks old females, 20-24 g, purchased from Charles Rivers, Wilmington). Tumor growth was tracked by regularly measuring the length and width of tumors with a caliper. The tumor volume $(V)$ was estimated by the mathematical ellipsoid formula:

$V=\left(\frac{\pi}{6}\right) \times(\text { width })^{2} \times($ length $)$

When tumors reached an average volume of $200 \mathrm{~mm}^{3}$, mice were adequately randomized and divided into three experimental groups. Once reaching the desired volume, animals were administered with $200 \mu \mathrm{l}$ of treatment by a single intravenous injection in lateral tail vein: physiological saline solution $(0.9 \% \mathrm{NaCl})$ as control, PASN-DCX nanocapsules $\left(20 \mathrm{mg} / \mathrm{kg}\right.$ mouse weight) and Taxotere ${ }^{\circledR}$ (20 $\mathrm{mg} / \mathrm{kg}$ mouse weight). Administration day was considered as day zero. After treatments, tumor growth was tracked by regularly measuring the length and width of tumors with a caliper.

Treated groups were also compared in terms of mean of survival times, in days, after treatment administration. The percentage increase in survival time (\% IST) was determined relative to the median or mean survival of untreated controls as presented in the following equation:

$\% \mathrm{IST}=\frac{\operatorname{Mean}_{\mathrm{T}}-\text { Mean }_{\mathrm{C}}}{\text { Mean }_{\mathrm{C}}} \times 100$

where Mean ${ }_{\mathrm{T}}$ was the mean of survival time of the treated group and Mean $\mathrm{C}_{\mathrm{C}}$ is the mean of the survival time of the control group (Allard et al., 2009).

\subsection{Statistical analysis}

Statistical analysis of the in vivo data was conducted by the nonparametric Kruskal-Wallis method followed by the Tuckey HSC multiple comparison test ( $p<0.05$ was considered to be statistically significant). For the estimation of the mean survival times, we used a censured model, Kaplan Meier analysis, according to which censure events, defined as deaths, were considered as both extreme tumor growth, $>10 \%$ of mouse weight and the end of the evaluation period, assuming that in both cases deaths occur before the next size control of the tumor. Statistical significance was calculated using the log-rank test (Mantel-Cox test).

SPSS software version 16.0 (SPSS Inc.) was used for that purpose. The different treatment groups were compared in terms of range, and mean survival time (days), long term survivor (\%) and increase in survival time (ISTmean\%).

\section{Results and discussion}

In this paper we present the in vivo proof of concept of the potential of PASN nanocapsules as drug delivery vehicles in cancer therapy. As described above, our group has previously developed PASN nanocapsules and shown their ability to improve the in vitro cytotoxic efficacy of DCX on NCI-H460 cancer cell line. PASN was selected on the basis of its biodegradability (Metselaar et al., 2003), long circulating properties (Romberg et al., 2007b), as well as its interesting probable targeting properties, taking into account the avidity of cancer tumor cells for asparagine (McCredie et al., 1973). The studies here presented include biodistribution and pharmacokinetics of PASN nanocapsules as well as the efficacy of DCX-loaded PASN nanocapsules, as nanomedicine prototype. 
Table 1

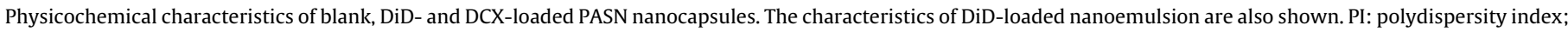
EE: encapsulation efficiency; DCX: Docetaxel; DiD: Fluorescent probe. Values are given as mean \pm SD; $n=3$.

\begin{tabular}{|c|c|c|c|c|}
\hline Formulation & Size $(\mathrm{nm})$ & PI & $\zeta$ potential $(\mathrm{mV})$ & $\mathrm{EE}(\%)$ \\
\hline Blank PASN nanocapsules & $187 \pm 7$ & 0.1 & $-38 \pm 3$ & - \\
\hline DiD-loaded nanoemulsion & $192 \pm 3$ & 0.1 & $-20 \pm 7$ & $60.3 \pm 5$ \\
\hline DiD-loaded PASN nanocapsules & $163 \pm 3$ & 0.1 & $-29 \pm 6$ & $74.4 \pm 4$ \\
\hline DCX-loaded PASN nanocapsules & $176 \pm 3$ & 0.1 & $-43 \pm 6$ & $90.1 \pm 3$ \\
\hline
\end{tabular}

Table 2

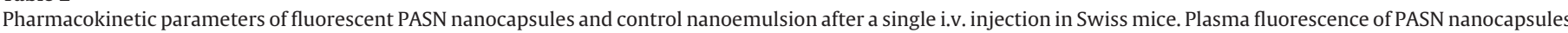
was measured over a $24 \mathrm{~h}$ period in animals treated with $1 \mathrm{mg}$ of $\mathrm{DiD} / \mathrm{kg}$ of mouse weight. Each data point represents the group mean $\pm \mathrm{SE}$.

\begin{tabular}{|c|c|c|c|c|}
\hline Formulation & $t_{1 / 2 \text { distribution }}(\mathrm{h})$ & $t_{1 / 2 \text { elimination }}(\mathrm{h})$ & $\operatorname{MRT}(\mathrm{h})$ & $\mathrm{AUC}_{0-24}(\mathrm{mg} / \mathrm{ml} \mathrm{h})$ \\
\hline PASN-nanocapsules & $0.58 \pm 0.1^{*}$ & $12.64 \pm 0.2^{*}$ & $12.75 \pm 0.4^{*}$ & $34.83 \pm 2.3^{*}$ \\
\hline Nanoemulsion & $0.34 \pm 0.3$ & $8.17 \pm 0.3$ & $6.59 \pm 0.1$ & $11.43 \pm 2.1$ \\
\hline
\end{tabular}

* Significant differences with respect to the nanoemulsion; $p<0.05$.

\subsection{Characterization of DiD-labeled and DCX-loaded PASN nanocapsules}

Two sets of nanocapsules containing either DiD or DCX were prepared according to the solvent displacement technique, and characterized for their size, polydispersity, zeta potential and encapsulation efficiency. The results presented in Table 1 indicate that PASN nanocapsules form homogeneous populations with a mean particle size of around $190 \mathrm{~nm}$ and a negative surface charge (around $-40 \mathrm{mV}$ ). The encapsulation efficiency of both hydrophobic molecules, DCX and DiD, was high (90\% and $74 \%$ respectively), with final concentrations in isolated formulations of around $1.8 \mathrm{mg} / \mathrm{ml}$ and $80 \mu \mathrm{g} / \mathrm{ml}$, respectively. The encapsulation of DCX did not significantly affect either the size or the zeta potential of the blank nanocapsules. However, the encapsulation of the fluorescent probe $\mathrm{DiD}$, led to a small reduction in both size and surface charge intensity. Nevertheless, these minor differences (13 nm-size and $14 \mathrm{mV}$-zeta) between the drug-loaded and fluorescent probeloaded nanocapsules are expected to be irrelevant with regard to their biodistribution.

\subsubsection{Hemolytic activity of PASN nanocapsules}

A key parameter for the acceptability of a formulation for i.v. administration, is the hemolytic activity. Drug-induced hemolysis in vitro is considered to be a simple and reliable estimation of membrane damage caused by drugs in vivo (Nornoo et al., 2008). The release of hemoglobin was used to quantify the membranedamaging properties of the PASN nanocapsules. To set the one hundred and zero percent values we used Triton X-100 and PBStreated erythrocytes, respectively. Erythrocytes were incubated for $1 \mathrm{~h}$ with five different nanocapsule concentrations (from $1 \mathrm{mg} / \mathrm{ml}$ to $10 \mathrm{mg} / \mathrm{ml}$ of PASN nanocapsules in blood), which corresponded to nanocapsules-blood dilutions of 1:1, 1:2, 1:5, 1:10 and 1:20 (Fig. 1). Under these conditions PASN nanocapsules showed no hemolytic effects, thus indicating no detectable disturbance of the red blood cell membranes. These positive results persuaded us to move ahead toward the pharmacokinetic evaluation of the blank nanocapsules.

\subsection{Pharmacokinetics and biodistribution of PASN nanocapsules}

For the study of the pharmacokinetics of PASN nanocapsules, we used fluorescent DiD-labeled nanocapsules injected in the tail vein of Swiss mice at a probe dose of $1 \mathrm{mg} / \mathrm{kg}$ of animal weight. Afterwards, blood samples were collected from $30 \mathrm{~min}$ to $24 \mathrm{~h}$, and the plasma was assessed for its fluorescent content. The fluorescence of a mixture of labeled nanocapsules with blood was taken as the $100 \%$ of the injected dose. Fig. 2 illustrates the plasma pharmacokinetic behavior of the fluorescent nanocapsules and also that of a fluorescent anionic nanoemulsion used as a control. Following the administration of the control nanoemulsion, a very rapid decrease in the fluorescence concentration was observed reaching the value of $15 \%$ of total fluorescence after $30 \mathrm{~min}$. On the contrary, the fluorescence associated to the nanocapsules remained at high concentrations (about 40\% of the initial dose) for at least $3 \mathrm{~h}$ post-injection. Finally, approximately $10 \%$ of the injected dose of

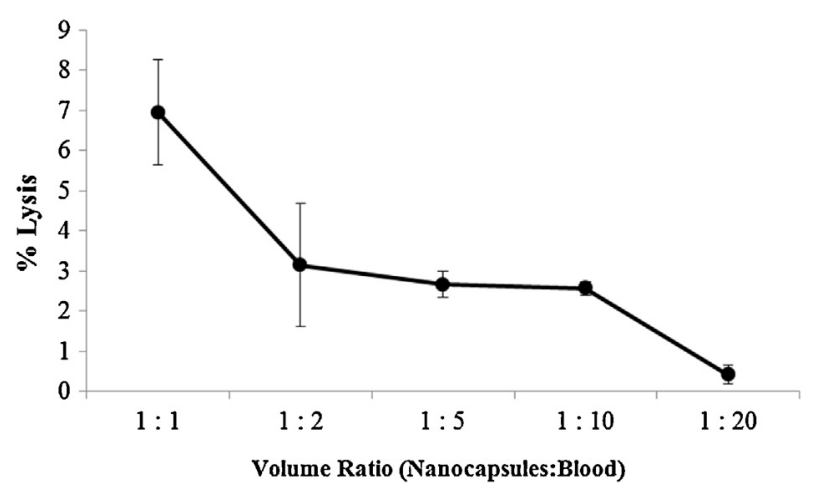

Fig. 1. Percentage of hemolytic activity $v s$ concentration of PASN nanocapsules after incubation for $1 \mathrm{~h}$. Cells were incubated at $37^{\circ} \mathrm{C}$ with PASN blood concentrations in the range of $1-10 \mathrm{mg} / \mathrm{ml}$, which correspond to the blood dilution range of $1: 20-1: 1$.

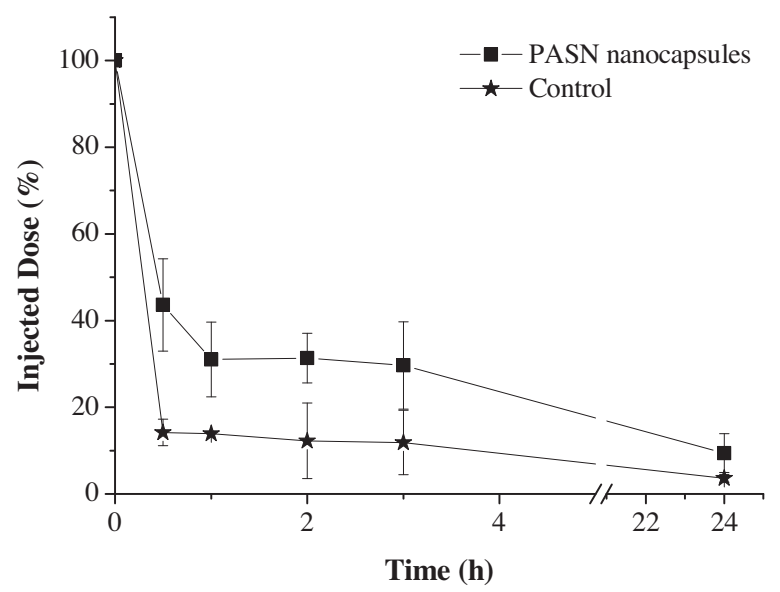

Fig. 2. Percentage of nanocarriers-associated fluorescence remaining in plasma after single bolus injection of PASN nanocapsules $(\mathbf{\square})$ in mice. The injected dose of DiD was $1 \mathrm{mg} / \mathrm{kg}$. The fluorescence observed upon administration of the control nanoemulsion $(\star)$ is also showed as a control. Each data point represents the group mean \pm SD of the percentage of injected dose. 
1h
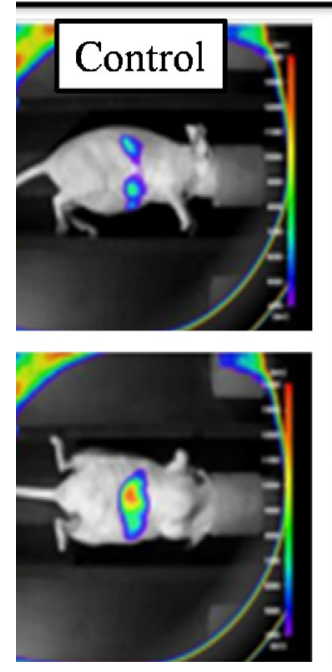
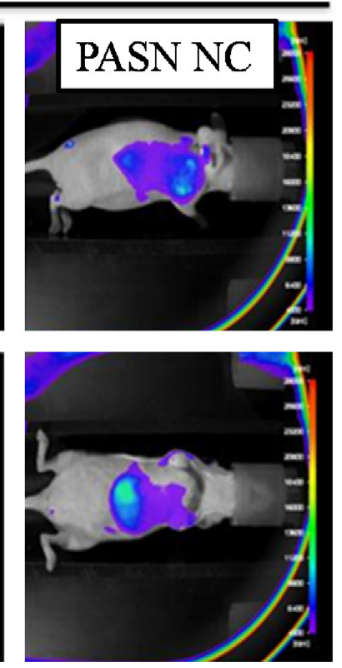

$24 h$
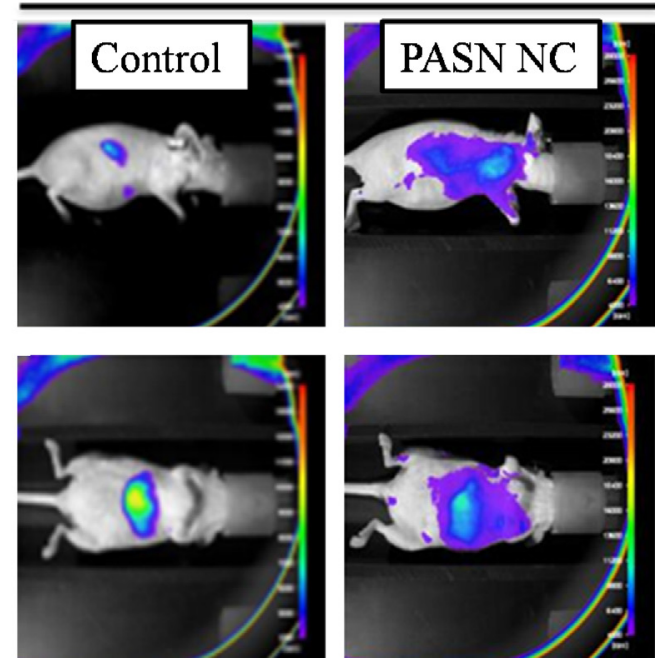

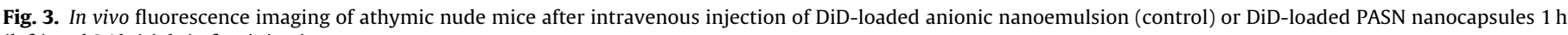
(left) and $24 \mathrm{~h}$ (right) after injection.

both anionic nanoemulsion and PASN nanocapsules remained in circulation $24 \mathrm{~h}$ after the injection.

A more illustrative way of presenting the plasma kinetics profiles of fluorescent nanocapsules is through their pharmacokinetic parameters. As shown in Table 2, PASN nanocapsules exhibited a 1.5 fold longer elimination half-life in respect to the control emul-

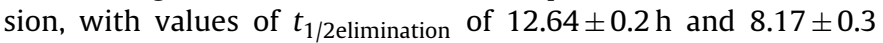
for PASN nanocapsules and anionic control nanoemulsion, respectively. On the other hand, the values of mean residence times (MRT) and area under the curve (AUC) were also significantly higher for the nanocapsules in comparison to the control emulsion. The MRT value of the nanocapsules was $12.75 \pm 0.4 \mathrm{~h}$, double than that of the control, while the AUC was extended around 200\% with respect to that of the anionic nanoemulsion (from $11.43 \pm 2.1 \mathrm{~h}$ to $34.83 \pm 2.3 \mathrm{~h})$.

Overall, the plasma kinetic profiles manifest the proof-ofconcept of the long-circulating properties of PASN nanocapsules. These results are in good agreement with those reported by Romberg et al. (Romberg et al., 2007c) with an analogous polymer to PASN used to coat liposomes. These authors showed that poly-hidroxyethyl-ASN coating provided a better protection of nanocarriers to plasmatic proteins and MPS than PEG coating. Even though it would not be reasonable to conclude from these studies the superiority of PASN vs PEG in terms of nanocarrier protection ability, we could at least state that the use of PASN coatings could be an interesting alternative technology for designing long-circulating nanocarriers. The superior AUC of DCX in PASN nanocapsules also refers to a major availability of the drug than could reach the tumor area and then interact closest with surface receptors of cells. Thus, as indicated above, the presence of a PASN corona on nanocapsules could result in their enhanced uptake by the cancer cells.

As a complementary study, the body distribution profile of the PASN nanocapsules and the control emulsion was tested in mice by monitoring the real-time NIR fluorescence intensity in the body. Near-infrared (NIR)-absorbing dyes represent a very interesting way to obtain information from living models since they can be monitored with safe, non-invasive optical imaging/contrasting techniques. The advantages of imaging in the NIR region are numerous: the significant reduction of background absorption, fluorescence and light scattering along with high sensitivity, the availability of low-cost sources of excitation and the versatility that allows the large catalog of existing probes.

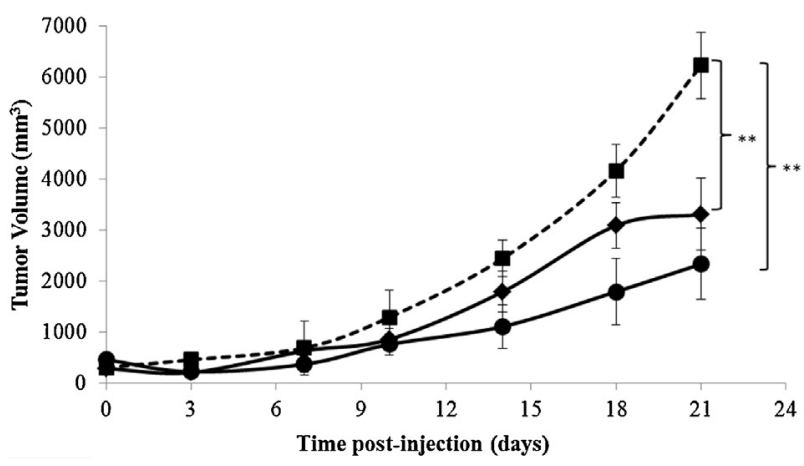

Fig. 4. Antitumor effect of DCX loaded-PASN nanocapsules $(\bullet)$ and Taxotere ${ }^{\circledR}(\bullet$ after a single intravenous administration as compared to control group receiving $0.9 \% \mathrm{NaCl}$ solution (serum) ( $\mathbf{\square}$ ) in a subcutaneous U87MG glioma mouse model. Statistical analysis was done at day 18 and 21 for DCX-loaded PASN nanocapsules and Taxotere ${ }^{\circledR}$ treatments compared to serum-receiving animals. ${ }^{* *} p<0.01$. All data are reported as mean $\pm \mathrm{SE} ; n=6$.

In accordance with the results of calculated pharmacokinetic parameters (Fig. 2 and Table 2), the nanoemulsion was rapidly eliminated from the blood compartment and localized in the liver and kidneys. In contrast, as noted by the diffused large NIR signal observed following administration of PASN nanocapsules, a significant amount of fluorescence remained in circulation for at least $24 \mathrm{~h}$. The prolonged circulation time showed by PASN nanocapsules is in accordance with that reported by other authors about the interesting stealth properties of PASN and its derivatives (Romberg et al., 2007b, 2007c) (Fig. 3).

\subsection{In vivo antitumor activity of DCX-loaded PASN nanocapsules}

U87MG subcutaneous glioma mouse model was used to evaluate the tumor reduction efficacy of DCX-loaded PASN nanocapsules. This activity was compared with those of commercial DCX form, Taxotere ${ }^{\circledR}$. Both treatments were administered when tumors reached $200 \mathrm{~mm}^{3}$. A third control group received an injection of saline solution (serum).

A dose of $20 \mathrm{mg}$ of DCX/kg of body weight was administered via i.v., in the vein tail of tumor-bearing mice, for each treatment in volumes not higher than $200 \mu$ l. The results presented in Fig. 4 show the tumor growth during the experiment after receiving different 
Table 3

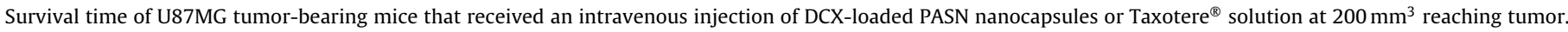
Mice injected with physiological serum were used as control group.

\begin{tabular}{|c|c|c|c|c|}
\hline \multirow[t]{2}{*}{ Treatment } & \multicolumn{2}{|c|}{ Mean survival time (days) } & \multicolumn{2}{|c|}{ Increased survival time (IST) (\%) } \\
\hline & Survival time range & Mean \pm SD & $\mathrm{IST}_{\text {mean }}$ & $p$-Value $v s$ serum group \\
\hline DCX-loaded PASN nanocapsules & $14-21$ & $18.7 \pm 2$ & 67 & 0.036 \\
\hline Taxotere $^{\circledR}$ & $11-21$ & $16.8 \pm 3$ & 50 & 0.056 \\
\hline Serum & $6-14$ & $11.2 \pm 2$ & - & \\
\hline
\end{tabular}

\% IST percentage of increase in survival time relative to that of the serum control group.

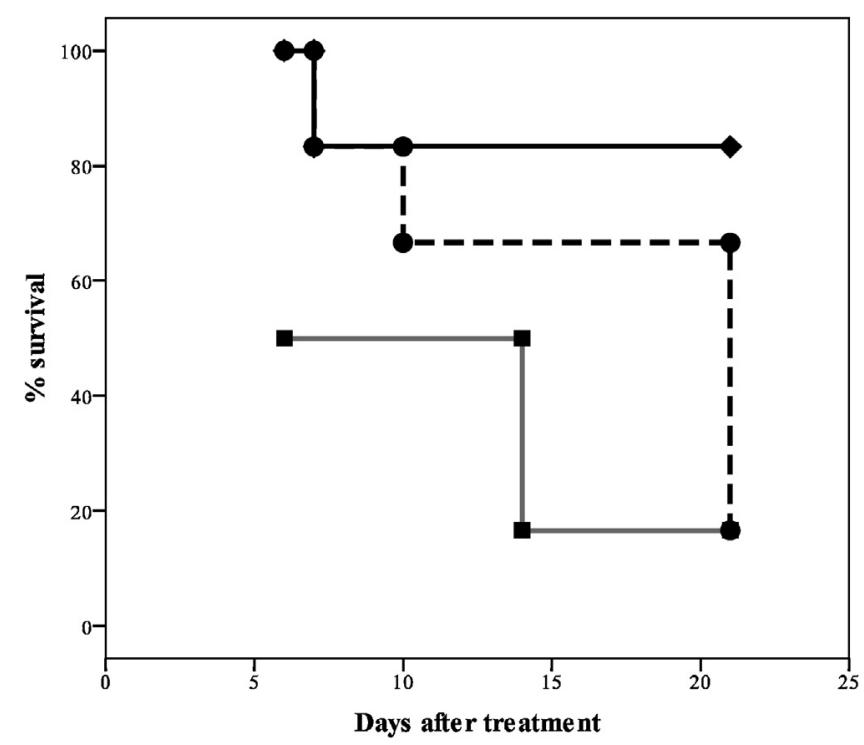

Fig. 5. Kaplan-Meier survival plots of subcutaneous U87MG glioma tumor-bearing mice following treatment with Taxotere ${ }^{\circledR}(\bullet)$ or DCX-loaded PASN nanocapsules $(\downarrow)$. Serum-treated mice were used as a control (ם).

treatments. Subcutaneous tumor in control groups receiving serum grew very quickly, reaching a relative tumor volume of about $6 \times 10^{3} \mathrm{~mm}^{3}$ after 21 days. On the contrary, the intravenous administration of both DCX-loaded PASN nanocapsules and Taxotere ${ }^{\circledR}$ significantly reduced the final volumes of the tumor as compared to the control group $(p<0.01)$.

As a complement of the antitumor activity study of DCX-loaded nanocapsules, we evaluated the survival rates in the three studied groups. Kaplan-Meier survival plots are displayed in Fig. 5 For the calculation of the mean survival times, a censored model was used, which takes as a censure event an excessive tumor volume (more than $10 \%$ of mouse weight), the final of experiment or dead. The obtained results show that the PASN nanocapsules treatment improves significantly $(p<0.05)$ the survival rate of mice treated (Fig. 5, Table 3). In fact PASN nanocapsules and Taxotere ${ }^{\circledR}$ increased the mean survival time in a $67 \%$ and $50 \%$, respectively relative to the control.

Taking into account the efficacy results as well as those of the plasma kinetic profiles, we could speculate on a more adequate biodistribution profile for the nanocapsules compared to the commercial formulation. The improved biodistribution profile would imply a facilitated accumulation of the drug in the tumor tissue and a reduced accumulation in other vital organs. This targeting capacity of these novel nanocarriers could involve a simple passive targeting similar to that reported for a variety of PEGylated nanocarriers loaded with taxanes (Yang et al., 2007; Crosasso et al., 2000; Danhier et al., 2009; Senthilkumar et al., 2008). However, we cannot discard the possibility for the PASN coating in providing a certain active targeting capacity based on the avidity of cancer cells for the ASN amino acid (Allan et al., 1967).

\section{Conclusion}

Here, we present the first proof-of-principle of in vivo efficacy of PASN nanocapsules as nanovehicles for anticancer drugs such as docetaxel. PASN nanocapsules exhibited a prolonged blood circulation and reduced accumulation in liver and kidneys. Also, the treatment with PASN nanocapsules containing docetaxel of U87MG glioma-bearing mice obtained a significant improvement over the survival rates compared to Taxotere ${ }^{\circledR}$, and an important reduction of tumor growth compared to control.

\section{Acknowledgements}

This work was supported by the European Commission FP7 EraNet - EuroNanoMed Program - Instituto de Salud Carlos III (Lymphotarg proyect, Ref. PS09/02670), and the Xunta de Galicia (Competitive Reference Groups-FEDER funds Ref. 2010/18). Giovanna Lollo acknowledges a fellowship from the Spanish Ministry of Education. Gustavo Rivera acknowledges the CONACyT-Mexico's fellowship.

\section{References}

Allan, J.D., Ireland, J.T., Milner, J., Moss, A.D., 1967. L-Asparagine, L-asparaginase and cancer. Lancet 1, 682-686.

Allard, E., Huynh, N.T., Vessieres, A., Pigeon, P., Jaouen, G., Benoit, J.P., Passirani, C., 2009. Dose effect activity of ferrocifen-loaded lipid nanocapsules on a 9L-glioma model. Int. J. Pharm. 379, 317-323.

Alonso, M.J., Couvreur, P., 2012. Historical view of the design and development of nanocarriers for overcoming biological barriers. In: Nanostructured Biomaterials for Overcoming Biological Barriers. The Royal Society of Chemistry, pp. 3-36 (Chapter 1).

Calvo, P., Vila-Jato, J.L., Alonso, M.J., 1997. Evaluation of cationic polymer-coated nanocapsules as ocular drug carriers. Int. J. Pharm. 153, 41-50.

Chabner, B., Friedmann, A., 2006. Asparaginase. In: Chabner, B., Longo, D. (Eds.), Cancer Chemotherapy and Biotherapy: Principles and Practice. Lippincott Williams \& Wilkins, Philadelphia, p. 879.

Crosasso, P., Ceruti, M., Brusa, P., Arpicco, S., Dosio, F., Cattel, L., 2000. Preparation, characterization and properties of sterically stabilized paclitaxel-containing liposomes. J. Control. Release 63, 19-30.

Danhier, F., Lecouturier, N., Vroman, B., Jérôme, C., Marchand-Brynaert, J., Feron, O. Préat, V., 2009. Paclitaxel-loaded PEGylated PLGA-based nanoparticles: in vitro and in vivo evaluation. J. Control. Release 133, 11-17.

Garcion, E., Lamprecht, A., Heurtault, B., Paillard, A., Aubert-Pouessel, A., Denizot B., Menei, P., Benoit, J.P., 2006. A new generation of anticancer, drug-loaded, colloidal vectors reverses multidrug resistance in glioma and reduces tumor progression in rats. Mol. Cancer Ther. 5, 1710-1722.

Hervella, P., Lozano, V., Garcia-Fuentes, M., Alonso, M.J., 2008. Nanomedicine: new challenges, opportunities in cancer therapy. J. Biomed. Nanotechnol. 4, 276-292.

Huynh, N.T., Roger, E., Lautram, N., Benoit, J.P., Passirani, C., 2010. The rise and rise of stealth nanocarriers for cancer therapy: passive versus active targeting. Nanomedicine 5, 1415-1433.

Lee, S.H., Yoo, S.D., Lee, K.H., 1999. Rapid and sensitive determination of paclitaxel in mouse plasma by high-performance liquid chromatography. J. Chromatogr. B: Analyt. Technol. Biomed. Life Sci. 724, 357-363.

Li, Y., Wang, J., Wientjes, M.G., Au, J.L., 2012. Delivery of nanomedicines to extracellular and intracellular compartments of a solid tumor. Adv. Drug Deliv. Rev. 64, 29-39.

Liu, Z., Jiao, Y., Wang, Y., Zhou, C., Zhang, Z., 2008. Polysaccharides-based nanoparticles as drug delivery systems. Adv. Drug Deliv. Rev. 60 1650-1662.

Lozano, M.V., Torrecilla, D., Torres, D., Vidal, A., Dominguez, F., Alonso, M.J., 2008. Highly efficient system to deliver taxanes into tumor cells: docetaxel-loaded chitosan oligomer colloidal carriers. Biomacromolecules 9, 2186-2193. 
McCredie, K.B., Ho, D.H.W., Freireich, E.J., 1973. L-Asparaginase for the treatment of cancer. CA Cancer J. Clin. 23, 220-227.

Metselaar, J.M., Bruin, P., De Boer, L.W.T., De Vringer, T., Snel, C., Oussoren, C., Wauben, M.H.M., Crommelin, D.J.A., Storm, G., Hennink, W.E., 2003. A novel family of L-amino acid-based biodegradable polymer-lipid conjugates for the development of long-circulating liposomes with effective drug-targeting capacity. Bioconjug. Chem. 14, 1156-1164.

Nornoo, A.O., Osborne, D.W., Chow, D.S.L., 2008. Cremophor-free intravenous microemulsions for paclitaxel: I: Formulation, cytotoxicity and hemolysis. Int. J. Pharm. 349, 108-116.

Oyarzun-Ampuero, F.A., Rivera-Rodríguez, G.R., Alonso, M.J., Torres, D., 2013. Hyaluronan nanocapsules as a new vehicle for intracellular drug delivery. Eur. J. Pharm. Sci., 483-490.

Rivera-Rodriguez, G.R., Alonso, M.J., Torres, D., 2013. Poly-L-asparagine nanocapsules as anticancer drug delivery vehicles. Eur. J. Pharm. Biopharm. (in press).

Romberg, B., Kettenes-Van Den Bosch, J.J., De Vringer, T., Storm, G., Hennink, W.E., 2006. 1H NMR spectroscopy as a tool for determining the composition of poly(hydroxyethyl-L-asparagine)-coated liposomes. Bioconjug. Chem. $17,860-864$

Romberg, B., Metselaar, J.M., Baranyi, L., Snel, C.J., Bünger, R., Hennink, W.E., Szebeni, J., Storm, G., 2007a. Poly(amino acid)s: promising enzymatically degradable stealth coatings for liposomes. Int. J. Pharm. 331, $186-189$
Romberg, B., Oussoren, C., Snel, C.J., Hennink, W.E., Storm, G., 2007b. Effect of liposome characteristics and dose on the pharmacokinetics of liposomes coated with poly(amino acid)s. Pharm. Res. 24, 2394-2401.

Romberg, B., Oussoren, C., Snel, C.J., Carstens, M.G., Hennink, W.E., Storm, G. 2007c. Pharmacokinetics of poly(hydroxyethyl-L-asparagine)-coated liposomes is superior over that of PEG-coated liposomes at low lipid dose and upon repeated administration. Biochim. Biophys. Acta 1768, 737-743.

Senthilkumar, M., Mishra, P., Jain, N.K., 2008. Long circulating PEGylated poly(D,Llactide-co-glycolide) nanoparticulate delivery of docetaxel to solid tumors. J. Drug Target. 16, 424-435.

Verma, N., Kumar, K., Kaur, G., Anand, S., 2007. L-Asparaginase: a promising chemotherapeutic agent. Crit. Rev. Biotechnol. 27, 45-62.

Wadhwa, S., Jain, A., Woodward, J.G., Mumper, R.J., 2012. Lipid nanocapsule as vaccine carriers for his-tagged proteins: evaluation of antigen-specific immune responses to HIV I His-Gag p41 and systemic inflammatory responses. Eur. J. Pharm. Biopharm. 80, 315-322.

Wang, A.Z., Langer, R., Farokhzad, O.C., 2012. Nanoparticle delivery of cancer drugs. Annu. Rev. Med. 63, 185-198.

Yang, T., Cui, F.-D., Choi, M.-K., Cho, J.-W., Chung, S.-J., Shim, C.-K., Kim, D.-D., 2007. Enhanced solubility and stability of PEGylated liposomal paclitaxel: in vitro and in vivo evaluation. Int. J. Pharm. 338, 317-326.

Yu, M.K., Park, J., Jon, S., 2012. Targeting strategies for multifunctional nanoparticles in cancer imaging and therapy. Theranostics 2, 3-44. 